\title{
Different molecular patterns in glioblastoma multiforme subtypes upon recurrence
}

\author{
Ramon Martinez • Veit Rohde - Gabriele Schackert
}

Received: 13 April 2009/Accepted: 6 July 2009/Published online: 31 July 2009

(c) The Author(s) 2009. This article is published with open access at Springerlink.com

\begin{abstract}
One of the hallmarks of glioblastoma is its inherent tendency to recur. At this point patients with relapsed GBM show a survival time of only few months. The molecular basis of the recurrence process in GBM is still poorly understood. The aim of the present study was to investigate the genetic profile of relapsed GBM compared to their respective primary tumors. We have included 20 paired GBMs. In all tumor samples, we have analyzed $p 53$ and $P T E N$ status by sequencing analysis, EGFR amplification by semiquantitative PCR and a wide-genome fingerprinting was performed by microsatellite analysis. Among primary GBM, we observed twelve type 2 GBM, four type 1 GBM and four further GBM showing neither $p 53$ mutations nor EGFR amplification (non-type 1-non-type 2 GBM). Upon recurrence, we have detected two molecular patterns of tumor progression: GBM initially showing either type 1 or type 2 profiles conserved them at the time of relapse. In contrast, non-type 1-non-type 2 GBM acquired the typical pattern of type 2 GBM and harbor EGFR amplification without $p 53$ mutation. New PTEN mutations upon relapse were only detected in type 2 GBM. Additional LOH were more frequently identified in relapses of type $2 \mathrm{GBM}$ than in those showing the type 1 signature. Taken together, our results strongly suggest that recurrences of GBM may
\end{abstract}

Electronic supplementary material The online version of this article (doi:10.1007/s11060-009-9967-4) contains supplementary material, which is available to authorized users.

R. Martinez $(\bowtie) \cdot$ V. Rohde

Department of Neurosurgery, University of Göttingen,

Robert-Koch-Str. 40, 37075 Göttingen, Germany

e-mail: ramon.martinez@med.uni-goettingen.de

G. Schackert

Department of Neurosurgery, University of Dresden,

Fetscherstr. 74, 01307 Dresden, Germany display two distinct pattern of accumulation of molecular alterations depending on the profile of the original tumor.

Keywords Glioblastoma - Relapse $\cdot$ p53 - PTEN . Microsatellite $\cdot$ Mutation

$\begin{array}{ll}\text { Abbreviations } \\ \text { GBM } & \text { Glioblastoma multiforme } \\ \text { TTR } & \text { Time to tumor relapse } \\ \text { MGMT } & O^{6} \text {-methyl-guanine-methyl-transferase } \\ \text { CFTR } & \text { Cystic fibrosis transmembrane regulator } \\ \text { KPS } & \text { Karnofsky performance score }\end{array}$

\section{Introduction}

Glioblastoma multiforme is the most frequent primary brain tumor in adults. One of its characteristic features is the intrinsic tendency to recur despite aggressive therapy [1]. Upon recurrence, the median survival of patients is only few months [2] whereas the overall median survival time is 15 months. This has not significantly changed in the last 20 years despite of advances in surgery, radio- und chemotherapy [3, 4] A moderate improvement of the 2-year survival rate has been achieved after therapy with the alkylating drug temozolomide in GBM with hypermethylated MGMT compared to those with unmethylated gene $(46 \%$ vs. $26 \%)[3,5,6]$.

GBM is one of the most intensively investigated human malignancy but the molecular mechanisms associated with recurrence are still poorly understood. Saxena et al. [7] reported a high rate of homozygous deletion of the genes $p 16^{I N K 4}$ and $p 15^{I N K 4 a}$ in GBM relapses. In a further report, 
this author investigated $10 \mathrm{GBM}$ and their corresponding relapses and observed that recurrences displayed the pattern of genetic alterations frequently observed in the de novo pathway (GBM without a low grade precursor lesion) [8]. Hulsebos et al. [9] analyzed 12 match paired GBM and found in relapse cases new $\mathrm{LOH}$ at chromosome regions $1 \mathrm{p} 36,19 \mathrm{q} 13,10 \mathrm{q} 23$ and $1 \mathrm{q} 25$. In contrast to the former study, GBM relapses encompassing new $p 53$ mutation or $E G F R$ amplification were not observed.

The identification of molecular features associated with recurrences of GBM is of major importance, since a better understanding of this process might provide clues for the development of efficient treatments. In the present analysis, we have examined the molecular signatures of forty tumors consisting in relapsed GBM and their corresponding primary neoplasms which had undergone surgery and radiochemotherapy. This included the status of $p 53$, PTEN and $E G F R$ as well as a wide genome $\mathrm{LOH}$ analysis with thirteen highly informative microsatellite markers at chromosome regions 17p13, 10q23, 1p35-36, 19q13, 13q14, 9p21.

\section{Materials and methods}

Patient population and tumor samples

All patients had undergone surgery with the goal of maximal possible tumor resection followed by fractionated radiotherapy (mean dose: 58 Gray) and chemotherapy. Forty paired tumors from twenty patients were available. Informed consent for samples and data analysis from each patient or the patient's caretaker was obtained.

Tumor samples were immediately frozen in liquid nitrogen after surgical resection and stored at $-80^{\circ} \mathrm{C}$. Tumor tissue was evaluated by experienced pathologists according to the $2000 \mathrm{WHO}$ classification criteria. DNA from tumor specimens was isolated applying the QIAamp ${ }^{\circledR}$ DNA Mini Kit (Qiagen, Hilden, Germany).

\section{LOH analysis}

LOH of chromosome 10q was studied with markers covering reported deletions on 10q23-24: D10S215, D10S541 and the intragenic PTEN marker PTENCA. Analysis of $\mathrm{LOH}$ at $17 \mathrm{p} 13$ was performed with a $p 53$ intragenic localized marker. Allelic losses at 9p21 $\left(p 16^{I N K 4 a}, p 14^{A R F}\right.$ and $p 15)$ and $13 \mathrm{q} 14(R B 1)$ were studied with primers D9S171, D9S1748, D9S1749, D13S153 and D13S267. Loss of heterozygosity at $1 \mathrm{p} 35-\mathrm{p} 36$ and $19 \mathrm{q} 13$ was assessed with the markers D1S468, D1S482, D19S112 and D19S412. Amplified PCR products were analyzed on a denaturing 6.5\% Long Ranger polyacrylamide gel on an Automated Laser Fluorescence sequencing device and analyzed using the ALLELELINKS ${ }^{\circledR} 1.00$ software (Amersham Pharmacia Biotech, Freiburg, Germany). Evaluation of LOH was performed as described [10].

\section{Semiquantitative PCR-analysis}

For studying amplification of EGFR a differential duplexPCR with the CFTR gene marker was carried out, as described [11]. Briefly, we calculated the EGFR/CFTR ratios $[x]$ from peripheral blood DNA of 20 healthy Caucasian adults. A value $[x+3 \mathrm{SD}]$ was considered as the cut-off level for the normal gene copy number. Ratios higher than $[x+3 \mathrm{SD}]$ were regarded as evidence of more than 2 copies of the EGFR gene.

Sequencing analysis of p53 and PTEN

Mutational analysis of $p 53$ and PTEN were performed in the DNA from both tumors and corresponding leukocytes. After amplifying all exons and intron-exon boundaries, we analyzed the PCR products on $1 \%$ agarose gel and excised and eluted them in sterile $\mathrm{H}_{2} \mathrm{O}$. Subsequently, they were subjected to cycle sequencing reactions using the Thermo Sequenase ${ }^{\circledR}$ Fluorescent Cycle Sequencing kit (Amersham Pharmacia Biotech). The cycle sequencing products were resolved using a denaturing 6.5\% Long Ranger polyacrylamide gel on a sequencing device. PTEN primer sequences and PCR conditions were previously described [12]. P53 primer sequences and PCR conditions are based on genome database entries (http://www.ncbi.nlm.nih.gov). Briefly, PCR reactions were carried out using a mixture containing 150 ng DNA, $10 \mathrm{mM}$ Tris, $2 \mathrm{mM} \mathrm{MgCl}_{2}, \mathrm{HCl}(\mathrm{pH} 8.3$ ), $0.2 \mathrm{mM}$ dNTP, $10 \mathrm{mM}$ concentrations of each primer and 0.5 unit of Taq DNA Polymerase. Temperature cycles and times for PCR reactions were: denaturation at $94^{\circ} \mathrm{C}$ for $30 \mathrm{~s}$, annealing at $65^{\circ} \mathrm{C}$ for $30 \mathrm{~s}$, and extension at $72^{\circ} \mathrm{C}$ for $30 \mathrm{~s}$. Each PCR reaction was preceded by a 3-min denaturation at $94^{\circ} \mathrm{C}$, and the final cycle was followed by a 3 -min extension at $72^{\circ} \mathrm{C}$. The total number of cycles for PCR amplification was $25-30$, depending on the sample DNA.

Statistical analysis

Student $t$-test, Chi-square test, Fisher's exact test and Mann-Whitney U-test, were performed to compare differences between groups depending on the analyzed variables. Spearman correlation test was used to evaluate the correlation between two parameters. Confidence interval (CI) was obtained through logistic regression. Survival studies were performed with the Kaplan Meier analysis and the log-rank test. A value of $P<0.05$ was considered to be significant. Analyses were performed with the SPSS software (version 10, SPSS Inc., Chicago, IL, USA). 


\section{Results}

Clinico-pathological characteristics of the patients

The male/female ratio was $1: 1$. The median age at diagnosis of primary tumor was 59.5 years (range: 27-69; SD: 12.25). The median time to tumor recurrence was 7.5 months (range: 3-24 months; SD: 5.1 ; 95\%-CI: $5.3-$ 7.4 months). With one only exception, all patients displayed at diagnosis a Karnofsky performance score (KPS) of $>70$ points. An association between age or KPS and TTR was not evidenced $(P=0.35, \rho=0.30$, Spearman correlation test). There were no significant associations between mutation of $p 53$ or $E G F R$ amplification and either survival time ( $P=0.250$ and 0.127 , respectively, log-rank test and Kaplan-Meier) or time to tumor relapse $(P=0.210$ and 0.287 , respectively, log-rank test and Kaplan-Meier).

Genetic analyses

In primary GBM we have observed $E G F R$ amplification without mutations of $p 53$ in 12 cases, thus indicating a type II GBM profile. Four further cases showed $p 53$ mutations (Table 1, Fig. 1) in the absence of EGFR amplification, which was indicative of a type 1 GBM signature. In four

Table 1 Sequence variations of $p 53$ in type 1 glioblastomas

\begin{tabular}{|c|c|c|c|c|c|}
\hline \multicolumn{6}{|c|}{ p53 sequence variations } \\
\hline Case & Sequence variation & Base exchange & $\begin{array}{l}\text { Aminoacid } \\
\text { substitution }\end{array}$ & CpG site & Distribution \\
\hline \multirow[t]{2}{*}{ G3p } & c. $278 \mathrm{C}>\mathrm{T}$ & $\mathrm{CCT} \rightarrow \mathrm{TCT}$ & Pro $\rightarrow$ Ser & No & $\begin{array}{l}\text { Somatic mutations in } 74 \text { tumors } \\
\text { Germline mut. in } 2 \text { Li-Fraumeni }{ }^{\text {a }}\end{array}$ \\
\hline & c. $266 \mathrm{G}>\mathrm{T}$ & $\mathrm{GGA} \rightarrow \mathrm{GTA}$ & $\mathrm{Gly} \rightarrow$ Val & No & $\begin{array}{l}\text { Somatic mutations in } 44 \text { tumors } \\
\text { No germline mutations }{ }^{\mathrm{a}}\end{array}$ \\
\hline \multirow[t]{2}{*}{$\mathrm{G} 3 \mathrm{r}$} & c. $278 \mathrm{C}>\mathrm{T}$ & $\mathrm{CCT} \rightarrow \mathrm{TCT}$ & Pro $\rightarrow$ Ser & No & $\begin{array}{l}\text { Somatic mutations in } 74 \text { tumors } \\
\text { Germline mut. in } 2 \mathrm{Li} \text {-Fraumeni }{ }^{\text {a }}\end{array}$ \\
\hline & c. $266 \mathrm{G}>\mathrm{T}$ & $\mathrm{GGA} \rightarrow \mathrm{GTA}$ & $\mathrm{Gly} \rightarrow \mathrm{Val}$ & No & $\begin{array}{l}\text { Somatic mutations in } 44 \text { tumors } \\
\text { No germline mutations }{ }^{\mathrm{a}}\end{array}$ \\
\hline \multirow[t]{3}{*}{ G7p } & c. $72 \mathrm{C}>\mathrm{G}$ (Arg72Pro) & $\mathrm{CCC} \rightarrow \mathrm{CGC}$ & Arg $\rightarrow$ Pro & No & $\begin{array}{l}\text { Validated polymorphism } \\
\text { Higher risk lung cancer [34] }\end{array}$ \\
\hline & IVS3+21_37dup (P53PIN3) & & & & $\begin{array}{l}\text { Validated polymorphism } \\
\text { Higher risk of CRC [36] }\end{array}$ \\
\hline & c. $278 \mathrm{C}>\mathrm{T}$ & $\mathrm{CCT} \rightarrow \mathrm{TCT}$ & Pro $\rightarrow$ Ser & No & $\begin{array}{l}\text { Somatic mutations in } 74 \text { tumors } \\
\text { Germline mut. in } 2 \text { Li-Fraumeni }^{\text {a }}\end{array}$ \\
\hline \multirow[t]{3}{*}{ G7r } & c.72C>G (Arg72Pro) & $\mathrm{CCC} \rightarrow \mathrm{CGC}$ & Arg $\rightarrow$ Pro & No & $\begin{array}{l}\text { Validated polymorphism } \\
\text { Higher risk lung cancer [34] }\end{array}$ \\
\hline & IVS3+21_37dup (P53PIN3) & & & & $\begin{array}{l}\text { Validated polymorphism } \\
{ }^{\mathrm{a}} \text { Higher risk of CRC [36] }\end{array}$ \\
\hline & c. $278 \mathrm{C}>\mathrm{T}$ & $\mathrm{CCT} \rightarrow \mathrm{TCT}$ & Pro $\rightarrow$ Ser & No & $\begin{array}{l}\text { Somatic mutations in } 74 \text { tumors } \\
\text { Germline mut. in } 2 \text { Li-Fraumeni }{ }^{\text {a }}\end{array}$ \\
\hline G14p & $\begin{array}{l}\text { c. } 14 \mathrm{G}>\mathrm{C} \\
\text { c. } 273 \mathrm{G}>\mathrm{A}\end{array}$ & $\begin{array}{l}\mathrm{CTG} \rightarrow \mathrm{CTC} \\
\mathrm{CGT} \rightarrow \mathrm{CAT}\end{array}$ & $\begin{array}{l}\text { Leu } \rightarrow \text { Leu } \\
\text { Arg } \rightarrow \text { His }\end{array}$ & $\begin{array}{l}\text { No } \\
\text { Yes }\end{array}$ & $\begin{array}{l}\text { Not described mutation } \\
\text { Somatic mutations in } 733 \text { tumors } \\
\text { Germline mut. in } 16 \text { Li-Fraumeni }^{\text {a }}\end{array}$ \\
\hline G14r & $\begin{array}{l}\text { c. } 14 \mathrm{G}>\mathrm{C} \\
\text { c. } 273 \mathrm{G}>\mathrm{A}\end{array}$ & $\begin{array}{l}\mathrm{CTG} \rightarrow \mathrm{CTC} \\
\mathrm{CGT} \rightarrow \mathrm{CAT}\end{array}$ & $\begin{array}{l}\text { Leu } \rightarrow \text { Leu } \\
\text { Arg } \rightarrow \text { His }\end{array}$ & $\begin{array}{l}\text { No } \\
\text { Yes }\end{array}$ & $\begin{array}{l}\text { Not described mutation } \\
\text { Somatic mutations in } 733 \text { tumors } \\
\text { Germline mut. in } 16 \text { Li-Fraumeni }^{\text {a }}\end{array}$ \\
\hline \multirow[t]{2}{*}{ G16p } & c. $273 \mathrm{G}>\mathrm{A}$ & $\mathrm{CGT} \rightarrow \mathrm{CAT}$ & $\mathrm{Arg} \rightarrow \mathrm{His}$ & Yes & $\begin{array}{l}\text { Somatic mutations in } 733 \text { tumors } \\
\text { Germline mut. in } 16 \text { Li-Fraumeni }^{\text {a }}\end{array}$ \\
\hline & IVS2+38G $>C$ & & & & Validated polymorphism [41] \\
\hline \multirow[t]{2}{*}{ G16r } & c. $273 \mathrm{G}>\mathrm{A}$ & $\mathrm{CGT} \rightarrow \mathrm{CAT}$ & Arg $\rightarrow$ His & Yes & $\begin{array}{l}\text { Somatic mutations in } 733 \text { tumors } \\
\text { Germline mut. in } 16 \text { Li-Fraumeni }^{\text {a }}\end{array}$ \\
\hline & IVS2+38G $>C$ & & & & Validated polymorphism [41] \\
\hline
\end{tabular}

${ }^{a}$ According to $p 53$ data bases entries, mut mutation, $C R C$ colorectal cancer 
Fig. 1 Upper row: wild type sequence of p53. Lower row: base exchange mutation (GGA $\rightarrow$ GTA) of $p 53$ (exon 8 , codon 266), predicting the aminoacid substitution Gly $\rightarrow$ Val. The arrow indicates the base exchange

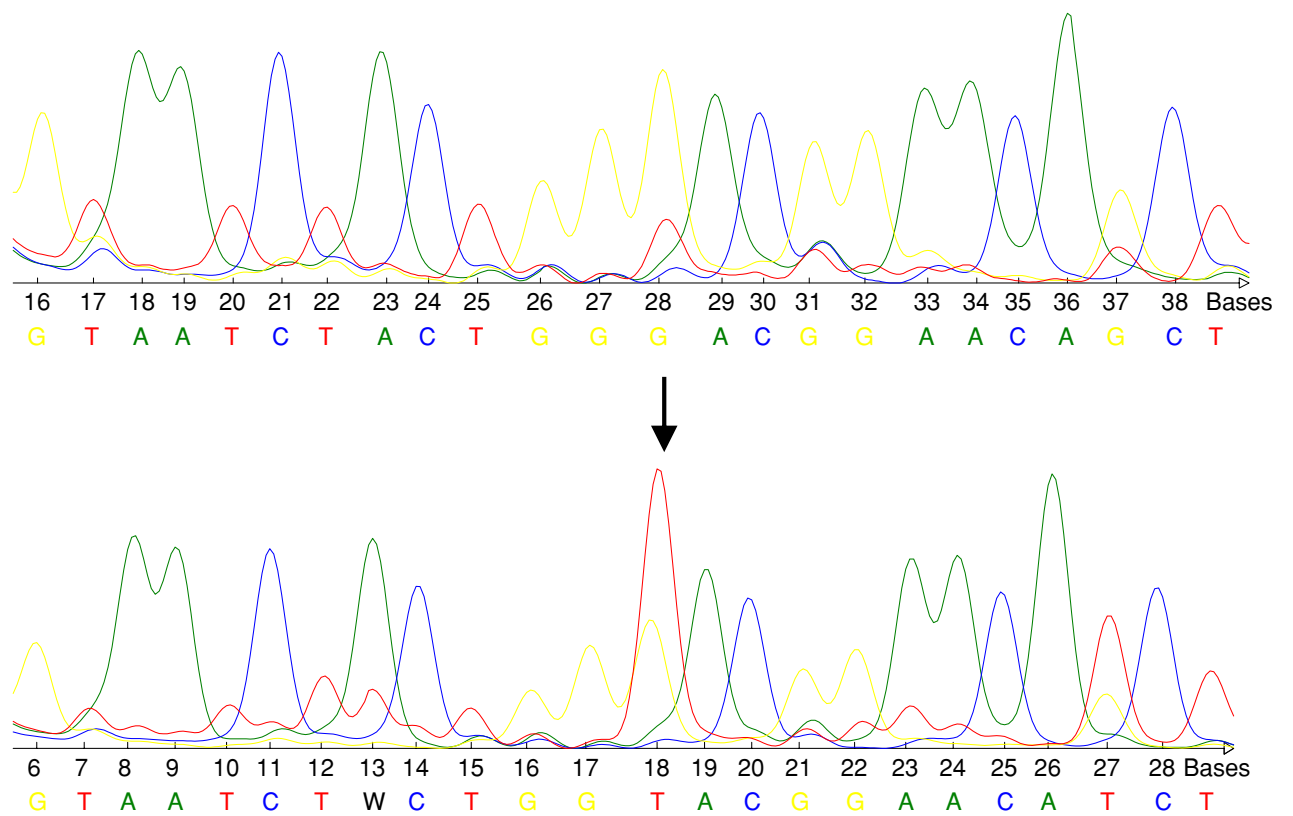

GBM neither $p 53$ mutations nor EGFR amplification were identified (non-type 1-non-type 2 GBM).

At the time of recurrence, every type $1 \mathrm{GBM}$ and type 2 GBM conserved their genetic pattern concerning p53 mutational status and EGFR amplification. On the other hand, relapses of non-type 1-non-type 2 GBM showed in all cases a new EGFR amplification, thus acquiring the signature of type $2 \mathrm{GBM}$. Additional PTEN mutations in relapse cases were observed only among type 2 GBM (Table 2). Overall, additional LOH were observed more frequently in relapses of type $2 \mathrm{GBM}(83.3 \%)$, than in those of type $1(50 \%)$ or non-type 1-non-type 2 tumors $(25 \%)$. Further results are shown in Table 3.

Among p53 mutations there were one $\mathrm{C}: \mathrm{G}$ to $\mathrm{A}: \mathrm{T}$ transition mutation at $\mathrm{CpG}$ site (codon 273), one $\mathrm{C}>\mathrm{T}$ transition at non-CpG site (codon 278), two transversion mutations at non- $\mathrm{CpG}$ sites $(\mathrm{G}>\mathrm{C}$ at codon 14 and $\mathrm{G}>\mathrm{T}$ at codon 266, Fig. 1) as well as one validated polymorphism in exon $4(\mathrm{G}>\mathrm{C}$ at codon 72). Moreover, we have observed two validated intronic polymorphisms in intron 3 (IVS3+21_37dup) and intron 2 (IVS2+38G $>$ C). One of the mutations (c.14G $>C$ ) has not been previously described in p53 data bases (http://www.p53.iarc.fr, http://www.p53. free.fr). A detailed characterization of $p 53$ sequence variations is shown in Table 1.

Concerning PTEN mutations, we have identified four transition mutations in exons 2, 5, 6 and 7, in all cases predicting aminoacid substitution. Furthermore, there was an acceptor site splice mutation at the conserved junction nucleotides (IVS8-2A>G). Finally, we have observed two transversion mutations in exon $5(\mathrm{c} .105 \mathrm{G}>\mathrm{T})$ and exon 4 (c.75C $>$ A, Fig. 2) as well as four intronic polymorphisms (Table 2). The transition in exon $6($ c.177A $>\mathrm{G})$, the splicing mutation and two of the polymorphisms have not been previously reported (Table 2 ).

The results of EGFR amplification analysis and widegenome LOH are shown in Table 3 and Supplementary Table 1 (EGFR/CFTR ratios). Interestingly, GBM cases harboring mutations of $p 53$ always displayed $\mathrm{LOH}$ at 17 p13 as well. Similarly, tumors featuring PTEN mutations showed $\mathrm{LOH}$ at $10 \mathrm{q} 23$, at least in one of the microsatellites used to analyze this region.

\section{Discussion}

GBM mostly exceed in its occurrence and mortality beyond any other primary brain tumor in adults [13]. On molecular level, two subsets of GBM have been recognized on the basis of genetic make-up and clinical features [14, 15]. Type 2 (de novo) GBM occurs commonly in elderly patients and exhibit overexpression and/or amplification of EGFR. Type 1 (secondary) GBM shows typically histological progression from a previous low grade astrocytoma and affects young patients. Paradigmatically, mutations of p53 will be observed (for review see [15]). Most of GBM cases belong to the type 2 group and genetic events are mutually exclusive for $E G F R$ and $p 53$. Moreover, there are GBM without either EGFR amplification or $p 53$ mutations (non-type 1-non-type $2 \mathrm{GBM}$ ) [16, 17]. During the past decade, accumulated evidence pointed to that type 1 and type 2 GBM constitute distinct disease entities developing through different genetic pathways [13, 14], showing different RNA and protein patterns $[18,19]$ and also probably differing in response to treatment [15]. 
Table 2 Sequence variations of PTEN in first and relapse glioblastomas

\begin{tabular}{|c|c|c|c|c|}
\hline \multicolumn{5}{|c|}{ PTEN sequence variations } \\
\hline Case & Sequence variation & Mutation effect & Aminoacid substitution & Distribution \\
\hline \multirow[t]{2}{*}{ G1p } & c. $44 \mathrm{C}>\mathrm{T}$ & $\mathrm{GGC} \rightarrow \mathrm{GGT}$ & Gly $\rightarrow$ Gly & Bladder cancer [39] \\
\hline & c. $105 \mathrm{G}>\mathrm{T}$ & $\mathrm{TGT} \rightarrow \mathrm{TTT}$ & Cys $\rightarrow$ Phe & Gliomas [38] \\
\hline \multirow[t]{2}{*}{ G1r } & c. $44 \mathrm{C}>\mathrm{T}$ & $\mathrm{GGC} \rightarrow \mathrm{GGT}$ & Gly $\rightarrow$ Gly & Bladder cancer [39] \\
\hline & c. $105 \mathrm{G}>\mathrm{T}$ & $\mathrm{TGT} \rightarrow \mathrm{TTT}$ & Cys $\rightarrow$ Phe & Gliomas [38] \\
\hline \multirow[t]{3}{*}{ G2p } & c. $177 \mathrm{~A}>\mathrm{G}$ & $\mathrm{TAT} \rightarrow \mathrm{TGT}$ & Tyr $\rightarrow$ Cys & Not described mutation \\
\hline & IVS $8+32 \mathrm{G}>\mathrm{T}$ & & & Validated polymorphism \\
\hline & & & & Cowden/Bannayan-Riley syndromes [40] \\
\hline \multirow[t]{3}{*}{$\mathrm{G} 2 \mathrm{r}$} & c. $177 \mathrm{~A}>\mathrm{G}$ & $\mathrm{TAT} \rightarrow \mathrm{TGT}$ & $\mathrm{Tyr} \rightarrow$ Cys & Not described mutation \\
\hline & IVS $8+32 \mathrm{G}>\mathrm{T}$ & & & Validated polymorphism \\
\hline & & & & Cowden/Bannayan-Riley syndromes [40] \\
\hline \multirow[t]{3}{*}{ G4 } & c. $105 \mathrm{G}>\mathrm{T}$ & $\mathrm{TGT} \rightarrow \mathrm{TTT}$ & Cys $\rightarrow$ Phe & Gliomas [38] \\
\hline & IVS $8+32 \mathrm{G}>\mathrm{T}$ & & & Validated polymorphism \\
\hline & & & & Cowden/Bannayan-Riley syndromes [40] \\
\hline \multirow[t]{3}{*}{ G4r } & c. $105 \mathrm{G}>\mathrm{T}$ & $\mathrm{TGT} \rightarrow \mathrm{TTT}$ & Cys $\rightarrow$ Phe & Gliomas [38] \\
\hline & IVS $8+32 \mathrm{G}>\mathrm{T}$ & & & Validated polymorphism \\
\hline & & & & Cowden/Bannayan-Riley syndromes [40] \\
\hline \multirow[t]{2}{*}{ G5p } & IVS5-28_29ins C & & & Not described polymorphism \\
\hline & IVS2-96A $>G$ & & & Not described polymorphism \\
\hline \multirow[t]{2}{*}{ G5r } & IVS5-28_29ins C & & & Not described polymorphism \\
\hline & c. $88 \mathrm{~A}>\mathrm{G}$ & $\mathrm{TAT} \rightarrow \mathrm{TGT}$ & Tyr $\rightarrow$ Cys & Head neck squamous cell cancer [42] \\
\hline G11p & IVS5-28_29ins C & & & Not described polymorphism \\
\hline \multirow[t]{3}{*}{ G11r } & IVS5-28_29ins C & & & Not described polymorphism \\
\hline & c. $88 \mathrm{~A}>\mathrm{G}$ & $\mathrm{TAT} \rightarrow \mathrm{TGT}$ & Tyr $\rightarrow$ Cys & Head \& neck squamous cell cancer [42] \\
\hline & IVS8-2A>G & Splice mutation & & Not described mutation \\
\hline G13p & IVS5-28_29ins C & & & Not described polymorphism \\
\hline \multirow[t]{3}{*}{ G13r } & IVS5-28_29ins C & & & Not described polymorphism \\
\hline & IVS8-2A>G & Splice mutation & & Not described mutation \\
\hline & c. $75 \mathrm{C}>\mathrm{A}$ & $\mathrm{CAT} \rightarrow \mathrm{AAT}$ & His $\rightarrow$ Asn & Not described mutation \\
\hline \multirow[t]{3}{*}{ G17p } & IVS4 109_110 ins TCTTA & & & Validated polymorphism \\
\hline & & & & Cowden/Bannayan-Riley syndromes [40] \\
\hline & c. $177 \mathrm{~A}>\mathrm{G}$ & $\mathrm{TAT} \rightarrow \mathrm{TGT}$ & Tyr $\rightarrow$ Cys & Not described mutation \\
\hline \multirow[t]{4}{*}{ G17r } & IVS4 109_110 ins TCTTA & & & Validated polymorphism \\
\hline & & & & Cowden/Bannayan-Riley syndromes [40] \\
\hline & c. $177 \mathrm{~A}>\mathrm{G}$ & $\mathrm{TAT} \rightarrow \mathrm{TGT}$ & Tyr $\rightarrow$ Cys & Not described mutation \\
\hline & IVS5-28_29ins C & & & Not described polymorphism \\
\hline \multirow[t]{2}{*}{ G19p } & IVS $8+32 \mathrm{G}>\mathrm{T}$ & & & Validated polymorphism \\
\hline & & & & Cowden/Bannayan-Riley syndromes [40] \\
\hline \multirow[t]{3}{*}{ G19r } & IVS $8+32 \mathrm{G}>\mathrm{T}$ & & & Validated polymorphism \\
\hline & & & & Cowden/Bannayan-Riley syndromes [40] \\
\hline & c. $252 \mathrm{~A}>\mathrm{G}$ & $\mathrm{GAT} \rightarrow \mathrm{GGT}$ & Asp $\rightarrow$ Gly & Glioma [43] \\
\hline
\end{tabular}

After multimodal therapy, GBM almost always recur. Although this is a capital feature of GBM, only few investigations with series of match paired patients are available from the literature and available data is partially contradictory $[7-9,20,21]$. We aimed to investigate whether molecular subtypes of GBM also show different profiles at the time of relapse.

In our genetic analysis, we have observed two patterns of tumor recurrence (Fig. 3): GBM type 1 and type 2 retained upon recurrence their genetic alterations affecting 
Table 3 Review of genetic changes of primary and relapsed glioblastomas

\begin{tabular}{|c|c|c|c|c|c|c|c|c|c|c|c|c|c|}
\hline Case & Sex & Age* $^{*}$ & Treatment & TTR & $\begin{array}{l}\text { Mut. } \\
\text { p53 }\end{array}$ & $\begin{array}{l}\text { Ampl. } \\
\text { EGFR }\end{array}$ & $\begin{array}{l}\text { Mut. } \\
\text { PTEN }\end{array}$ & \multicolumn{6}{|c|}{ Loss of heterozygosity (LOH) } \\
\hline & & & & & & & & 1 p35 & $9 p 21$ & $13 q 14$ & $19 q 13$ & $10 q 23$ & $17 \mathrm{p} 13$ \\
\hline G1p & $\mathrm{m}$ & 60 & no ther & 3 & $\mathrm{n}$ & $\mathrm{y}$ & $\mathrm{y}$ & & & & & & \\
\hline G1r & $\mathrm{m}$ & 60 & no ther & & $\mathrm{n}$ & $\mathrm{y}$ & $\mathrm{y}$ & & & & & & \\
\hline G2p & $\mathrm{m}$ & 60 & RT & 6 & $\mathrm{n}$ & $\mathrm{n}$ & $\mathrm{y}$ & & & & & & \\
\hline G2r & $\mathrm{m}$ & 60 & $A, V$ & & $\mathrm{n}$ & $\mathrm{y}$ & $\mathrm{y}$ & & & & & & \\
\hline G3p & $f$ & 62 & $\mathrm{RT}$ & 7 & $\mathrm{y}$ & $\mathrm{n}$ & $\mathrm{n}$ & & & & & & \\
\hline G3r & $f$ & 62 & $A, V$ & & $y$ & $\mathrm{n}$ & $\mathrm{n}$ & & & & & & \\
\hline G4p & $\mathrm{m}$ & 64 & $\mathrm{RT}$ & 3 & $n$ & $y$ & $y$ & & & & & & \\
\hline G4r & $\mathrm{m}$ & 64 & no ther & & $n$ & $\mathrm{y}$ & $\mathrm{y}$ & & & & & & \\
\hline G5p & $\mathrm{m}$ & 49 & $\mathrm{RT}$ & 9 & $n$ & $y$ & $n$ & & & & & & \\
\hline G5r & $\mathrm{m}$ & 49 & $A, V$ & & $n$ & $y$ & $y$ & & & & & & \\
\hline G6p & f & 57 & $\mathrm{RT} / \mathrm{A}, \mathrm{V}$ & 10 & $\mathrm{n}$ & $\mathrm{n}$ & $n$ & & & & & & \\
\hline G6r & $f$ & 58 & $A, V$ & & $n$ & $y$ & $n$ & & & & & & \\
\hline G7p & $f$ & 31 & no ther & 9 & $y$ & $\mathrm{n}$ & $n$ & & & & & & \\
\hline G7r & $f$ & 32 & $\mathrm{RT} / \mathrm{A}, \mathrm{V}$ & & $\mathrm{y}$ & $\mathrm{n}$ & $n$ & & & & & & \\
\hline G8p & $\mathrm{m}$ & 39 & $\mathrm{RT}$ & 12 & $n$ & $\mathrm{n}$ & $n$ & & & & & & \\
\hline G8r & $\mathrm{m}$ & 40 & no ther & & $n$ & $\mathrm{y}$ & $n$ & & & & & & \\
\hline G9p & $\mathrm{m}$ & 69 & $\mathrm{RT} / \mathrm{A}, \mathrm{V}$ & 16 & $n$ & $\mathrm{n}$ & $n$ & & & & & & \\
\hline G9r & $\mathrm{m}$ & 70 & $A, V$ & & $n$ & $y$ & $n$ & & & & & & \\
\hline G10p & $\mathrm{m}$ & 46 & $\mathrm{RT}$ & 17 & $n$ & $\mathrm{y}$ & $\mathrm{n}$ & & & & & & \\
\hline G10r & $\mathrm{m}$ & 47 & $A, V$ & & $n$ & $y$ & $n$ & & & & & & \\
\hline G11p & $\mathrm{m}$ & 37 & $\mathrm{RT}$ & 5 & $n$ & $\mathrm{y}$ & $\mathrm{n}$ & & & & & & \\
\hline G11r & $\mathrm{m}$ & 38 & no ther & & $n$ & $y$ & $y$ & & & & & & \\
\hline G12p & $f$ & 65 & RT & 6 & $n$ & $\mathrm{y}$ & $n$ & & & & & & \\
\hline G12r & f & 65 & $A, V$ & & $\mathrm{n}$ & $\mathrm{y}$ & $\mathrm{n}$ & & & & & & \\
\hline G13p & $f$ & 55 & $\mathrm{RT}$ & 8 & $n$ & $y$ & $n$ & & & & & & \\
\hline G13r & $f$ & 56 & no ther & & $n$ & $y$ & $y$ & & & & & & \\
\hline G14p & $f$ & 68 & $\mathrm{RT}$ & 9 & $\mathrm{y}$ & $\mathrm{n}$ & $n$ & & & & & & \\
\hline G14r & $f$ & 69 & $A, V$ & & $y$ & $\mathrm{n}$ & $n$ & & & & & & \\
\hline G15p & $f$ & 64 & $\mathrm{RT}$ & 8 & $n$ & $\mathrm{y}$ & $n$ & & & & & & \\
\hline G15r & $f$ & 65 & no ther & & $n$ & $\mathrm{y}$ & $n$ & & & & & & \\
\hline G16p & $f$ & 27 & RT & 24 & $\mathrm{y}$ & $\mathrm{n}$ & $n$ & & & & & & \\
\hline G16r & $f$ & 29 & $\mathrm{~T}$ & & $y$ & $\mathrm{n}$ & $n$ & & & & & & \\
\hline G17p & $\mathrm{m}$ & 61 & $\mathrm{RT}$ & 8 & $\mathrm{n}$ & $\mathrm{y}$ & y & & & & & & \\
\hline G17r & $\mathrm{m}$ & 62 & $A, V$ & & $n$ & $\mathrm{y}$ & $y$ & & & & & & \\
\hline G18p & $f$ & 59 & $\mathrm{RT} / \mathrm{T}$ & 12 & $n$ & $y$ & $n$ & & & & & & \\
\hline G18r & $f$ & 60 & $\mathrm{~T}$ & & $n$ & $\mathrm{y}$ & $n$ & & & & & & \\
\hline G19p & $\mathrm{m}$ & 67 & $\mathrm{RT} / \mathrm{T}$ & 9 & $\mathrm{n}$ & $\mathrm{y}$ & $\mathrm{n}$ & & & & & & \\
\hline G19r & $\mathrm{m}$ & 67 & $\mathrm{~T}$ & & $\mathrm{n}$ & $\mathrm{y}$ & $\mathrm{y}$ & & & & & & \\
\hline G20p & $f$ & 58 & $\mathrm{RT} / \mathrm{T}$ & 14 & $\mathrm{n}$ & $\mathrm{y}$ & $\mathrm{n}$ & & & & & & \\
\hline G20r & $f$ & 59 & $\mathrm{~T}$ & & $n$ & $y$ & $n$ & & & & & & \\
\hline
\end{tabular}

TTR time to tumor relapse (months), Mut mutation, Ampl amplification, $p$ primary (first tumor), $r$ relapse (first relapse), $m$ male, $f$ female, $n o$ ther no adjuvant therapy performed, $R T$ radiotherapy, $A$ ACNU (nimustine), $V$ VM26 (teniposide), $T$ temozolomide. Black boxes loss of heterozygosity, grey boxes retention of heterozygosity, white boxes not informative marker

* Age (years) at first operation

p53 and EGFR. In contrast, all relapses of non-type 1-nontype 2 GBM showed a new EGFR amplification, thus they acquired a type $2 \mathrm{GBM}$ profile. Accumulation of additional genetic alterations upon relapse included PTEN mutations in four type 2 GBM (but not in the other subtypes). Additional LOH were more frequently observed in relapses of type 2 GBM (in 10/12 cases) compared to relapses of type 1 GBM and non-type 1-non-type 2 GBM (Table 3). 

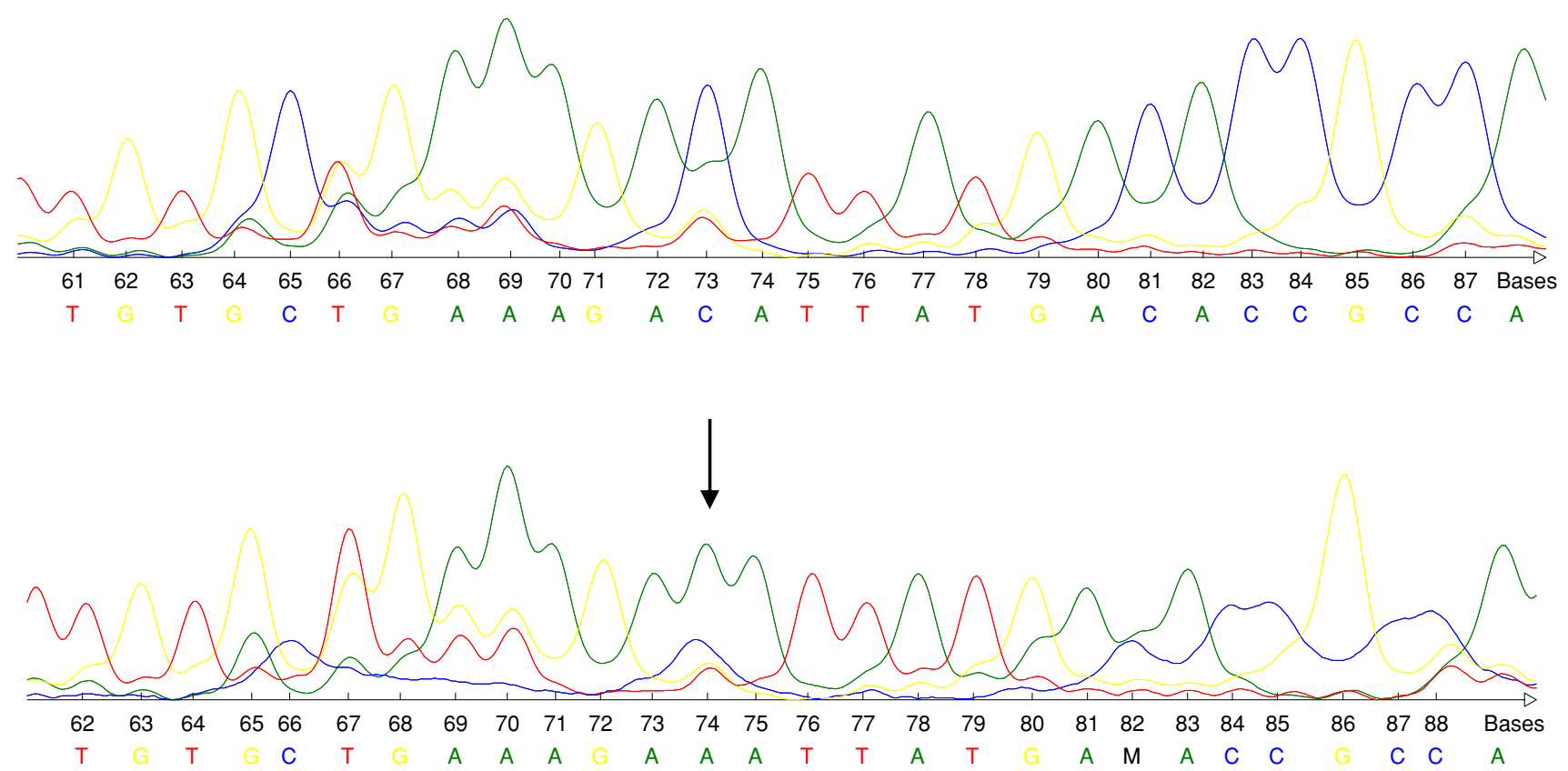

Fig. 2 Upper row: wild type sequence of PTEN. Lower row: base exchange mutation (CAT $\rightarrow$ AAT) of PTEN (exon 4, codon 75) predicting the aminoacid substitution His $\rightarrow$ Asn. The arrow indicates the base exchange

Fig. 3 Graphic showing two molecular patterns of GBM recurrence

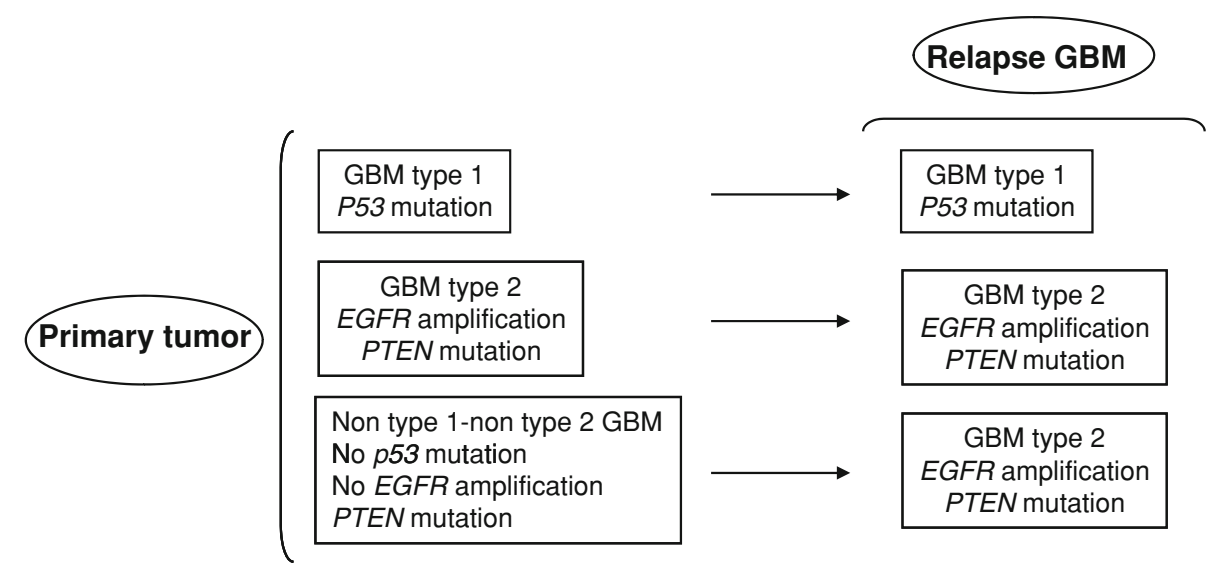

The accumulation of alterations during the clonal expansion of transformed glial cells is well known in GBM, confer tumor cells a growth advantage and underlies the genetic heterogeneity of GBM $[22,23]$. This phenomenon contributes to explain the molecular differences between primary tumors and relapses [9, 24, 25].

As a part of one previous investigation we had characterized the promoter methylation status of the DNA repair gene $M G M T$ in all type 1 GBM (Table 3, cases G3, G7, G14 and G16), non-type 1-non-type 2 GBM (Table 3, cases G2, G6, G8 and G9) as well as eight type 2 GBM here described [26]. This analysis together with the present results of $p 53$ and $E G F R$ have evidenced similar rates of $M G M T$ methylation for the three GBM subtypes, and they are in accordance with the overall methylation rates reported in glioblastoma [5, 27]. Typically, $M G M T$ was methylated in the first GBM and remained always methylated upon relapse. Thus, our results also confirmed the observation that, in the pathogenesis and progression of GBM, MGMT becomes hypermethylated at an early stage [28, 29] and remains methylated through progression, a situation which was has been described in small adenomas and carcinomas of colorectal origin as well [30]. The p53 mutational analysis in those type $1 \mathrm{GBM}$ with methylated MGMT showed G:C to A:T transition within $\mathrm{CpG}$ site (c.273 CGT $\rightarrow$ CAT) in two cases. Further mutations of $p 53$ in tumors with methylated MGMT (1 case) occurred in non-CpG sites. From p53 data bases entries, approximately $52 \%$ of mutational events are transition mutations and, of this subset, about $72 \%$ are G:C to A:T transitions (http:// www.p53.iarc.fr, http://www.p53.free.fr) [31], although the signature of the mutational spectrum varies according to 
tumor type. It was previously suggested a link [29] between MGMT methylation and the presence of $\mathrm{G}: \mathrm{C}$ to A:T transition mutations in $p 53$ in gliomas. In the present study we could confirm the former in two patients (cases G14p, G14r, G16p and G16r, Table 1); in these cases, G:C to A:T transitions could be attributable to a inactivation in $M G M T$ that allows $O^{6}$-methylguanine to persist and be read as an adenine. Our observations contrast with a recent investigation about GBM reporting only $4 \%$ of mutations of $p 53$ in tumors with methylated MGMT within CpG sites [32] although we should take in mind the size of our type 1 GBM collective, clearly too short to state a definite tendency.

Strikingly, we have identified in cases G7p and G7r the exonic p53 polymorphism c.72C $>\mathrm{G}$ (Arg72Pro) together with the intronic polymorphism IVS3+21_37dup (P53PIN3). Several investigations have pointed the association between $p 53$ polymorphisms and an increased risk for different cancers: for instance, in the case of the exonic c. 72 variant such association was suggested since the p53 protein with $\mathrm{Arg} 72$ is more efficient in inducing apoptosis than the one with the Pro72 variant [33]. In clinical studies, the variants c.72 Pro/Pro and c.72 Arg/Pro have been associated with an elevated risk of lung cancer [34, 35]. Recently, intron 3 duplication (IVS3+21_37dup) has been found to be associated with increased risk of colorectal cancer [36]. However, the authors could not determine whether this intron 3 duplication alone influences mRNA stability or if this effect requires the codon Pro72 variant. To our knowledge, the occurrence of both polymorphisms in GBM patients has not been reported before and pointed to a possible role in brain cancer as well.

With respect to PTEN, we have identified mutations only in type 2 GBM. In four cases, PTEN mutations occurred in both the first GBM and the relapse. In four further cases, new mutations were observed in the recurrences but not in the first tumor. The rate and pattern of mutations are in accordance with previous reported data [37]. All tumors with PTEN mutations also harbored $\mathrm{LOH}$ at the chromosome region $10 \mathrm{q} 23$, which pointed to the inactivation of this tumor suppressor gene and make patent its contribution to the tumorigenesis and progression in GBM.

Taken together, our results strongly suggest that GBM relapses, compared to the corresponding first tumor, accumulate additional molecular alterations and may develop along two distinct pathways, which contribute to delineate the different profiles of type 1, type 2 and nontype 1-non-type 2 GBM.

Open Access This article is distributed under the terms of the Creative Commons Attribution Noncommercial License which permits any noncommercial use, distribution, and reproduction in any medium, provided the original author(s) and source are credited.

\section{References}

1. Salcman M, Kaplan R (1991) Intracranial tumors in adults. In: Salcman M (ed) Neurology of brain tumors. Williams \& Wilkins, Baltimore, pp 1339-1352

2. Brandes AA, Ermani M, Basso U, Amistà P, Berti F, Scienza R, Rotilio A, Pinna G, Gardiman M, Monfardini S (2001) Temozolomide as a second-line systemic regimen in recurrent highgrade glioma: a phase II study. Ann Oncol 12:255-257

3. Stupp R, Dietrich PY, Ostermann-Kraljevic S, Pica A, Maillard I, Maeder P, Meuli R, Janzer R, Pizzolato G, Miralbell R, Porchet F, Regli L, de Tribolet N, Mirimanoff RO, Leyvraz S (2002) Promising survival for patients with newly diagnosed glioblastoma multiforme treated with concomitant radiation plus temozolomide followed by adjuvant temozolomide. J Clin Oncol 20:1375-1382

4. Walker MD, Green SB, Byar DP, Alexander E Jr, Batzdorf U, Brooks WH, Hunt WE, MacCarty CS, Mahaley MS Jr, Mealey J Jr, Owens G, Ransohoff J 2nd, Robertson JT, Shapiro WR, Smith KR Jr, Wilson CB, Strike TA (1980) Randomized comparisons of radiotherapy and nitrosoureas for the treatment of malignant glioma after surgery. N Engl J Med 303:1323-1329

5. Gorlia T, van den Bent MJ, Hegi ME, Mirimanoff RO, Weller M, Cairncross JG, Eisenhauer E, Belanger K, Brandes AA, Allgeier A, Lacombe D, Stupp R (2008) Nomograms for predicting survival of patients with newly diagnosed glioblastoma: prognostic factor analysis of EORTC and NCIC trial 26981-22981/CE.3. Lancet Oncol 9:29-38

6. Stupp R, Mason WP, van den Bent MJ, Weller M, Fisher B, Taphoorn MJ, Belanger K, Brandes AA, Marosi C, Bogdahn U, Curschmann J, Janzer RC, Ludwin SK, Gorlia T, Allgeier A, Lacombe D, Cairncross JG, Eisenhauer E, Mirimanoff RO, European Organisation for Research and Treatment of Cancer Brain Tumor and Radiotherapy Groups; National Cancer Institute of Canada Clinical Trials Group (2005) Radiotherapy plus concomitant and adjuvant temozolomide for glioblastoma. N Engl J Med 352: 987-996

7. Saxena A, Robertson JT, Ali IU (1996) Abnormalities of p16, p15 and CDK4 genes in recurrent malignant astrocytomas. Oncogene 13:661-664

8. Saxena A, Shriml LM, Dean M, Ali IU (1999) Comparative molecular genetic profiles of anaplastic astrocytomas/glioblastomas multiforme and their subsequent recurrences. Oncogene 18:1385-1390

9. Hulsebos TJ, Troost D, Leenstra S (2004) Molecular-genetic characterisation of gliomas that recur as same grade or higher grade tumours. J Neurol Neurosurg Psychiatry 75:723-726

10. Cawkwell L, Lewis FA, Quirke P (1994) Frequency of allele loss of DCC, p53, RB1, WT1, NF1, NM23 and APC/MCC in colorectal cancer assayed by fluorescent multiplex polymerase chain reaction. Br J Cancer 70:813-818

11. Hunter SB, Abbott K, Varma VA, Olson JJ, Barnett DW, James D (1995) Reliability of differential PCR for the detection of EGFR and MDM2 gene amplification in DNA extracted from FFPE glioma tissue. J Neuropathol Exp Neurol 54:57-64

12. Liaw D, Marsh DJ, Li J, Dahia PL, Wang SI, Zheng Z, Bose S, Call KM, Tsou HC, Peacocke M, Eng C, Parsons R (1997) Germline mutations of the PTEN gene in Cowden disease, an inherited breast and thyroid cancer syndrome. Nat Genet 16:64-67

13. Ohgaki H, Kleihues P (2005) Population-based studies on incidence, survival rates, and genetic alterations in astrocytic and oligodendroglial gliomas. J Neuropathol Exp Neurol 64:479-489

14. Ohgaki H, Dessen P, Jourde B, Horstmann S, Nishikawa T, Di Patre PL, Burkhard C, Schüler D, Probst-Hensch NM, Maiorka PC, Baeza N, Pisani P, Yonekawa Y, Yasargil MG, Lütolf UM, 
Kleihues P (2004) Genetic pathways to glioblastoma: a population-based study. Cancer Res 64:6892-6899

15. Ohgaki H, Kleihues $P$ (2007) Genetic pathways to primary and secondary glioblastoma. Am J Pathol 170:1445-1453

16. Benjamin R, Capparella J, Brown A (2003) Classification of glioblastoma multiforme in adults by molecular genetics. Cancer J 9:82-90

17. Lang F, Miller D, Koslow M, Newcomb E (1994) Pathways leading to glioblastoma multiforme: a molecular analysis of genetic alterations in 65 astrocytic tumors. J Neurosurg 81:427-436

18. Furuta M, Weil RJ, Vortmeyer AO, Huang S, Lei J, Huang TN, Lee YS, Bhowmick DA, Lubensky IA, Oldfield EH, Zhuang Z (2004) Protein patterns and proteins that identify subtypes of glioblastoma multiforme. Oncogene 23:6806-6814

19. Tso CL, Freije WA, Day A, Chen Z, Merriman B, Perlina A, Lee Y, Dia EQ, Yoshimoto K, Mischel PS, Liau LM, Cloughesy TF, Nelson SF (2006) Distinct transcription profiles of primary and secondary glioblastoma subgroups. Cancer Res 66:159-167

20. Spiegl-Kreinecker S, Pirker C, Marosi C, Buchroithner J, Pichler J, Silye R, Fischer J, Micksche M, Berger W (2007) Dynamics of chemosensitivity and chromosomal instability in recurrent glioblastoma. Br J Cancer 96:960-969

21. Stark AM, Witzel P, Strege RJ, Hugo HH, Mehdorn HM (2003) $\mathrm{p} 53, \mathrm{mdm} 2$, EGFR, and msh2 expression in paired initial and recurrent glioblastoma multiforme. J Neurol Neurosurg Psychiatry 74:779-783

22. Berkman RA, Clark WC, Saxena A, Robertson JT, Oldfield EH, Ali IU (1992) Clonal composition of glioblastoma multiforme. J Neurosurg 77:432-437

23. Morse RP, Darras BT, Ye Z, Wu JK (1994) Clonal analysis of human astrocytomas. J. Neurooncol 21:151-157

24. Grasbon-Frodl EM, Kreth FW, Ruiter M, Schnell O, Bise K, Felsberg J, Reifenberger G, Tonn JC, Kretzschmar HA (2007) Intratumoral homogeneity of MGMT promoter hypermethylation as demonstrated in serial stereotactic specimens from anaplastic astrocytomas and glioblastomas. Int J Cancer 121:2458-2464

25. Parkinson JF, Wheeler HR, Clarkson A, McKenzie CA, Biggs MT, Little NS, Cook RJ, Messina M, Robinson BG, McDonald KL (2008) Variation of $\mathrm{O}(6)$-methylguanine-DNA methyltransferase (MGMT) promoter methylation in serial samples in glioblastoma. J Neurooncol 87:71-78

26. Martínez R, Setien F, Voelter C, Casado S, Quesada MP, Schackert G, Esteller M (2007) CpG island promoter hypermethylation of the pro-apoptotic gene caspase- 8 is a common hallmark of relapsed glioblastoma multiforme. Carcinogenesis 28:1264-1268

27. Hegi ME, Diserens AC, Gorlia T, Hamou MF, de Tribolet N, Weller M, Kros JM, Hainfellner JA, Mason W, Mariani L, Bromberg JE, Hau P, Mirimanoff RO, Cairncross JG, Janzer RC, Stupp R (2005) MGMT gene silencing and benefit from temozolomide in glioblastoma. N Engl J Med 352:997-1003

28. Esteller M, Hamilton SR, Burger PC, Baylin SB, Herman JG (1999) Inactivation of the DNA repair gene O6-methylguanineDNA methyltransferase by promoter hypermethylation is a common event in primary human neoplasia. Cancer Res 59:793-797
29. Nakamura M, Watanabe T, Yonekawa Y, Kleihues P, Ohgaki H (2001) Promoter methylation of the DNA repair gene MGMT in astrocytomas is frequently associated with $\mathrm{G}: \mathrm{C} \rightarrow \mathrm{A}: \mathrm{T}$ mutations of the TP53 tumor suppressor gene. Carcinogenesis 22:17151719

30. Esteller M, Toyota M, Sanchez-Cespedes M, Capella G, Peinado MA, Watkins DN, Issa JP, Sidransky D, Baylin SB, Herman JG (2000) Inactivation of the DNA repair gene O6-methylguanineDNA methyltransferase by promoter hypermethylation is associated with $\mathrm{G}$ to A mutations in K-ras in colorectal tumorigenesis. Cancer Res 60:2368-2371

31. Greenblatt MS, Bennett WP, Hollstein M, Harris CC (1994) Mutations in the p53 tumor suppressor gene: clues to cancer etiology and molecular pathogenesis Cancer Res 54: 4855-4878

32. Cancer Genome Atlas Research Network (2008) Comprehensive genomic characterization defines human glioblastoma genes and core pathways. Nature 455:1061-1068

33. Dumont P, Leu JI, Della Pietra ACIII, George DL, Murphy M (2003) The codon 72 polymorphic variants of $\mathrm{p} 53$ have markedly different apoptotic potential. Nat Genet 33:357-365

34. Wu X, Zhao H, Amos CI, Shete S, Makan N, Hong WK, Kadlubar FF, Spitz MR (2002) p53 genotypes and haplotypes associated with lung cancer susceptibility and ethnicity. J Natl Cancer Inst 94:681-690

35. Fan R, Wu MT, Miller D, Wain JC, Kelsey KT, Wiencke JK, Christiani DC (2000) The p53 codon 72 polymorphism and lung cancer risk. Cancer Epidemiol Biomark Prev 9:1037-1042

36. Gemignani F, Moreno V, Landi S, Moullan N, Chabrier A, Gutiérrez-Enríquez S, Hall J, Guino E, Peinado MA, Capella G, Canzian F (2004) A TP53 polymorphism is associated with increased risk of colorectal cancer and with reduced levels of TP53 mRNA. Oncogene 23:1954-1956

37. Bonneau D, Longy M (2000) Mutations of the human PTEN gene. Hum Mutat 16: 109-122

38. Ishii N, Maier D, Merlo A, Tada M, Sawamura Y, Diserens AC, Van Meir EG (1999) Frequent co-alterations of TP53, p16/ CDKN2A, p14ARF, PTEN tumor suppressor genes in human glioma cell lines. Brain Pathol 9:469-479

39. Liu J, Babaian DC, Liebert M, Steck PA, Kagan J (2000) Inactivation of MMAC1 in bladder transitional-cell carcinoma cell lines and specimens. Mol Carcinog 29:143-150

40. Pezzolesi MG, Li Y, Zhou XP, Pilarski R, Shen L, Eng C (2006) Mutation-positive and mutation-negative patients with Cowden and Bannayan-Riley-Ruvalcaba syndromes associated with distinct 10q haplotypes. Am J Hum Genet 79:923-934

41. Pleasants LM, Hansen MF (1994) Identification of a polymorphism in intron 2 of the p53 gene. Hum Genet 93:607-608

42. Poetsch M, Lorenz G, Kleist B (2002) Detection of new PTEN/ MMAC1 mutations in head and neck squamous cell carcinomas with loss of chromosome 10. Cancer Genet Cytogenet 132:20-24

43. Zhang SJ, Endo S, Ichikawa T, Yoshimura J, Onda K, Tanaka R, Washiyama K, Kumanishi T (1999) Rare-type mutations of MMAC1 tumor suppressor gene in human glioma cell lines and their tumors of origin. Jpn J Cancer Res 90:934-941 